\title{
Are People Who Received Steroid Therapy Prone to Osteonecrosis of Femoral Head? A Systematic Review
}

\section{Wing Yi Pao ${ }^{1,2}$, Suzanne Sut Ying Chan $\mathrm{Ho}^{2}$, Zhao Min Liu ${ }^{2}$ and Ying Man Law ${ }^{3 *}$}

${ }^{1}$ Alice Ho Miu Ling Nethersole Charity Foundation, Nethersole Outreaching

Rehabilitation Mission, Hong Kong, Special Administrative Region, China

${ }^{2}$ The Chinese University of Hong Kong, The Jockey Club School of Public Health and

Primary Care, Hong Kong, Special Administrative Region, China

${ }^{3}$ The Hong Kong Polytechnic University, Department of Rehabilitation Sciences,

Hong Kong, Special Administrative Region, China

*Corresponding Author: Ying Man Law, The Hong Kong Polytechnic University, Department of Rehabilitation Sciences, Hong Kong, Special Administrative Region, China.
Received: February 25, 2021;

Published: March 10, 2021

(C) All rights are reserved by Ying Man Law., et al.

\section{Abstract}

The aim of this systematic review is to analyze the risk of developing osteonecrosis of femoral head (ONFH) for people received steroids therapy. Steroid has been considered as a risk factor of developing ONFH. However, there is no known systematic review that specifically focus on understanding steroid usage and the risk of developing ONFH. This review was conducted in accordance with the Preferred Reporting Items for Systematic Reviews and Meta-analyses (PRISMA) standards. Six electronic databases were searched: Cochrane, PubMed-MEDLINE, EMBASE, AMED, CINAHL and CJN. After eliminating duplicated articles and applying the inclusion criteria, five articles were selected for qualitative and the quantitative analysis: two case-control studies and three small sample cohort studies. The cohort studies indicate relative risk of the pooled data is 6.77 with $95 \% \mathrm{CI}(0.39,118.36)$. The casecontrol studies show a pooled odd ratio is 7.05 with $95 \% \mathrm{CI}(2.19,22.67)$. In general, findings shown to support that a higher risk of developing ONFH in people with steroid usage when compared with people without steroid usage. There are small number of related studies and their methodological quality is generally low. The risk of ONFH with steroids and the related dose-response relationship remain inconclusive from clinical studies. The unique clinical values of steroids should not be overlook due to the reference of the uncertain and unconfirmed research reports. Steroids remain its important values for certain patients; such as the infected with COVID-19 in the pandemic. Clinicians should administer steroids with cautions with careful assessment and monitoring to the patients. Whilst the possible risks and dose-response relationship cannot be confirmed from the related evidence, pharmological and nonpharmological adjuvant therapies should be prescribed together with steroids to minimize the potential risks of steroids therapy. Robust research is still needed to determine the suitable dosage and safe guidelines for using steroids to obtain the best therapeutic effects of it for the benefits of the patients in need.

Keywords: Avascular Necrosis; Coronavirus Disease 2019; Corticosteroid; COVID-19; Femoral Head; Necrosis; Osteonecrosis;

Pandemic; Risk; Steroid

Citation: Ying Man Law., et al. "Are People Who Received Steroid Therapy Prone to Osteonecrosis of Femoral Head? A Systematic Review". Acta Scientific Orthopaedics 4.4 (2021): 09-22. 


\section{Abbreviations}

CI: Confidence Interval; COVID-19: Coronavirus Disease 2019; MRI: Magnetic Resonance Imaging; NOS: Newcastle-Ottawa Scale; OR: Odd Ratio; ONFH: Osteonecrosis of Femoral Head; PRISMA: Preferred Reporting Items for Systematic Reviews and Meta-analyses; RCTs; Randomized Control Trials; RECOVERY: Randomized Evaluation of COVid-19 thERapY; RR: Risk Ratio; WHO: World Health Organization.

\section{Introduction}

The World Health Organization (WHO) recommends the use of systemic corticosteroid therapy for the patients with severe and critical Coronavirus Disease 2019 (COVID-19) and not to use systemic corticosteroids in the treatment of patients with non-severe COVID-19 based on the moderate certainty evidence of an important reduction in the risk of death [27]. Whilst specific vaccines, prevention and management of COVID-19 are still under extensive investigation, the use of steroids remains an important role in the pandemic for managing signs and symptoms of the disease, including the control of the inflammatory conditions resulted from COVID-19 infection. While the results from retrospective studies are heterogeneous and difficult to infer of a definitive protective benefit with corticosteroids, Randomized Evaluation of COVid-19 thERapY(RECOVERY) trial found a significantly better outcome with dexamethasone in severe cases. It reported a significant reduction of death by $35 \%$ in ventilated patients and by $20 \%$ amongst patients on supplemental oxygen therapy with the dexamethasone, although no benefit was observed in mild cases [20].

Corticosteroids, such as hydrocortisone and dexamethasone, have anti-inflammatory, antifibrotic, and vasoconstrictive effects. They are also known as steroids, which contain hormone cortisol, are chemicals that have a wide range of effects on the functions of the body and mainly used to relieve and regulate inflammation, carbohydrate metabolism, protein catabolism, blood electrolyte levels, and behaviours [16-17]. Studies reported that the use of steroids appear to be associated with benefit among critically ill patients with COVID-19 whether they are receiving mechanical ventilation or oxygen without mechanical ventilation [18]. However, the exact threshold at which an individual patient and the optimal dosage of corticosteroids prescription remain unclear. Potential side-effects from using corticosteroids include but not limit to stomach irrita- tion, indigestion, tachycardia, nausea, insomnia, muscle weakness, weight gain and osteoporosis [16].

Whilst clinical studies about the suitable type and dosage of steroids for the management of COVID-19 pandemic are heterogeneous and difficult to infer of a definitive protective benefit from corticosteroids, the possible adverse effects of steroids remain a concern in clinical practice. One of the possible side-effects of steroids is the development of avascular necrosis (or called osteonecrosis). This can cause chronic health conditions including bone damage, pain, disabilities and functional limitations in daily activities to the patients. Eventually, the patients' quality of life can be severely impaired due to the possible adverse effects of osteonecrosis from steroids therapy.

Osteonecrosis, which is also known as avascular necrosis or ischemic necrosis of the femoral head, is a pathologic process due to the interruption of blood supply to the involved bone area [1]. People with osteonecrosis of the head of femur often experience severe pain and thus affecting their mobility and causing dysfunction in daily activities. Mont., et al. (2010) [14] pointed out the high prevalence of clinical symptoms and femoral head collapse of untreated asymptomatic osteonecrosis of femoral head. Several risk factors, including alcohol consumption, steroid usage and/or smoking, are shown to be related to osteonecrosis of the femoral head $[7,12,14]$. It remains a concern that the improper use of systemic corticosteroids can increase the risk of having osteonecrosis of the femoral head to the patients [30].

Osteonecrosis of the femoral head (ONFH) is also known as avascular necrosis or ischemic necrosis of femoral head, which is commonly considered as a pathologic process due to the interruption of blood supply to the involved bone area [1,22]. In general, ONFH is a complex disease characterized by death of osteocytes and the bone marrow, and is caused by inadequate blood supply to the affected segment of the subchondral bone of the femoral head. The specific pathophysiology of steroid-induced ONFH is still unclear. ONFH is suggested to be the outcome of the combination of multiple factors and mechanisms. Several pathogenesis theories about possible steroid-induced ONFH are proposed including the lipid metabolism disorder theory, the decreased osteogenesis potential of bone marrow mesenchymal stem cells theory, insufficient blood supply theory, inflammation and cell apoptosis theory and 
gene polymorphism and non-coding RNA theory [25]. However, these theories have yet been confirmed. The exact pathogenesis about steroid-induced ONFH is still under investigation. With the advancement of technologies and precision research on stem cell and molecular biology, the theories and the pathologic process may be confirmed in the future. This will benefit to the design of suitable prevention and intervention to minimize and control the possible adverse effects of steroids including the development of ONFH.

Regardless to the underlying pathophysiological process, the management of osteonecrosis remains a perplexing problem in clinical practice. Surgical procedures are required for most symptomatic patients with collapse of the femoral head resulted from ONFH, such as transtrochanteric rotational osteotomy and curved varus osteotomy. The goals of osteotomy for ONFH are to avoid load stimulation on necrotic parts, and to return the femoral head from subluxation to concentric position for joint congruity [8]. Other surgical treatments such as joint-preserving or total hip arthroplasty are often needed for patients in later phases of the condition [15]. Without specific treatment $80 \%$ of clinically diagnosed cases will progress, and finally most cases will require arthroplasty [21].

Although steroids usage is widely considered as a risk factor of nontraumatic/idiopathic ONFH, the evidence on the topic remains anecdotal. Concerning to the risk of using steroid to the development of osteonecrosis, the estimation of the risk shows a wide range of differences in various studies. The risk of using steroids for the development of osteonecrosis of femoral head has not been systematically reviewed. Although low-dose ( $<1$ to $2 \mathrm{mg} / \mathrm{kg}$ ), shortterm (3-5 days) methylprednisolone was reported as an adjuvant treatment for COVID-19 [30], the dose-response relationship between the steroid therapy and the relative risk of ONFH remains unclear. There are only a few general overviews directly related to the risk factor.

However, most of the reported studies focus on qualitative reporting or have not conducted a complete systematic literature review on this specific topic. Although findings appeared that there is a potential risk of developing ONFH with the use of steroids, diversified results are identified with different dosages and clinical application to different populations in relevant studies. With the changes of using advanced technology for diagnosis, definition of the conditions, the context of the drug usage and increase in the amount of relevant studies from Asian countries since late 1980s; a review to update the knowledge in this area is needed. More research on the relevant topic may have been conducted which may contribute to a better estimation of the risk about the usage of steroids. There is no systematic review that we know of have specific focus on the steroid usage and the risk of ONFH. A clear under-standing on this specific topic is important for the clinical practice of evidence-based medicine for patients' benefits and safety.

Many questions remain unanswered about the use of steroids and the risk of ONFH. Will the people received steroids have a higher risk of developing ONFH compared with those who have not got such exposure in clinical practice? What do we know from clinical studies about risk of developing ONFH from the use of steroids? What is the potential dose-response relationship between the use of steroid and the risk of developing ONFH? Clinical guidelines for the prescription of steroids without causing possible consequence of developing ONFH has yet been well-determined. A systematic review provides specific understanding of this clinically important topic for clinical practice and further research.

Hence, the aim of this review is to systematically analyse the related clinical study reports particularly focus on the use of steroids and the risk of developing ONFH. This may provide specific and scientific information for supporting medical practitioners to prescribe steroids when necessary. The primary objective of this review is to summarize the best and updated evidence of the steroid usage as the risk factor of ONFH to people at all ages. The secondary objective is to critically review the study quality and methodological designs of the relevant primary clinical studies. This review also focus on the mean dosage of steroids to induce ONFH.

\section{Materials and Methods}

This review was conducted in accordance with the Preferred Reporting Items for Systematic Reviews and Meta-analyses (PRISMA) standards to evaluate the clinical studies and evidence of the effect about steroid usage to the risk of the ONFH. The main question of this review is: Are people at all ages who have had experience of steroid usage compared with those without using steroid at a higher risk of the development of osteonecrosis of femoral head?

Criteria for considering studies for this review Types of studies

Because of the limited or lack of RCTs on the risk of steroid usage, this review will therefore include cohort and case-control studies. 


\section{Types of participants}

Any persons at risk of development of ONFH, regardless of age, gender, race and health status, are included. Although the health status of participants is not controlled from the selection criteria, the information may be used to determine the need for sub-group analysis when heterogeneity of results noted.

\section{Types of interventions}

- In RCTs, at least one group should have received steroid as treatment with other comparison groups without steroid usage or received other treatments. Although it is un-common for RCTs to look for risk factors directly, some RCTs may still report ONFH as complications after steroid treatments.

- In cohort studies, the exposed group had received steroid as a treatment before the study or continuously within the study period, while the comparison group without such risk factor.

- In case-control studies, the case group had already developed osteonecrosis of femoral head while use of steroid would be one of the interested exposures in the studies. Case-control studies nested in cohort studies will be included if the study has described the main effect of steroid usage on ONFH.

\section{Types of outcome measures}

Primary outcome: the development of osteonecrosis of femoral head on one joint or both joints with diagnosis of osteonecrosis of femoral head according to anyone of the follows:

- World Health Organization (WHO) standard: ICD-10 (M8720) as ON induced by drugs.

- From previous literatures and medical manuals, such as "global hip score (i.e. Harris Hip Score, Mayo Score, Merle d'Aubigne-Postel Scores, etc.), hip revision, or the radiographic status" [15].

- The definition released in 1993 by the Association Research Circulation Osseous (ARCO) that "Bone necrosis as a disease that causes death of bone, and termed osteonecrosis." and the update by Sugano., et al., the Japan group of ARCO in 2002 that "Idiopathic osteonecrosis of femoral head is a disease that produces ischemic osteonecrosis of the femoral head without trauma or sepsis and progresses to secondary osteoarthritis after collapse of the femoral head."
- As the non-first-stage classification of ONFH in common classification systems mentioned in the study by Mont., et al. [14], including Classification system of Ficat and Arlet [4,23], Classification system of the University of Pennsylvania [21], Classification system of the Association Research Circulation Osseous [5] and Radiographic Classification system of the Japanese Orthopaedic Association [24].

- Having collapse of the joint or leading to operation (such as total hip arthroplasty).

\section{Searching methods for identification of relevant studies}

To comprehensively search to identify all studies relevant to the topic, no language restriction was imposed. Papers not in English were also considered. However, since MRI is the most accurate measure of diagnosis, only studies after 1985 (MRI starts to be used in human subject officially in 1977, but becomes more commonly use in osteonecrosis since approximately 1985) were included to ensure the quality of diagnosis method. The topic has many related animal studies, however, only human studies were considered based on the topic of this study.

\begin{tabular}{|l|l|}
\hline 1. & osteonecrosis.mp. \\
\hline 2. & avascular necrosis.mp. \\
\hline 3. & ischemic necrosis.mp. \\
\hline 4. & femoral head.mp. \\
\hline 5. & femoral head necrosis.mp. \\
\hline 6. & steroid*.mp. \\
\hline 7. & corticosteroid*.mp. \\
\hline 8. & glucocortcoid*.mp. \\
\hline 9. & cortisone*.mp. \\
\hline 10. & adrenal cortex hormone*.mp. \\
\hline 11. & risk*.mp. \\
\hline 12. & $\begin{array}{l}\text { exp osteonecrosis or exp femur head necrosis or } \\
\text { exp bone necrosis/ MeSH }\end{array}$ \\
\hline 13. & exp steroid/or exp corticosteroid/ MeSH \\
\hline 14. & 1 or 2 or 3 or 5 or 12 \\
\hline 15. & 4 and 14 \\
\hline 16. & 6 or 7 or 8 or 9 or 10 or 13 \\
\hline 17. & 15 and 16 \\
\hline 18. & 11 and 17 \\
\hline 19. & limit 18 to (human and yr="1985 -Current") \\
\hline
\end{tabular}

Table 1: Lists of vocabularies and text words for database searching. 


\section{Electronic searches}

Relevant studies were searched from the following six electronic databases: Cochrane Library, PubMed-MEDLINE, EMBASE, AMED, CINAHL and China Journal Net (CJN). The search covered the literature published up to October 2020. Sensitive search strategies using controlled vocabulary and text words were developed for each database based on items. The details are listed in Table 1 below. Since restriction may reduce the sensitivity in search, specific search strategy to filter cohort and case-control studies was not applied since these observational studies have not been previously labelled properly.

\section{Searching other resources}

The reference lists of all eligible primary studies and published reviews from electronic searching, which met all the selection criteria, were checked. Hand searching was also conducted to identify relevant studies. Key journals of epidemiology, orthopaedics and related topics about ONFH were also searched. Conference and seminar papers were also been searched for relevant contents. $\mathrm{Au}-$ thors of the identified primary studies were contacted by emails for missing details and to seek for additional information.

\section{Data collection and analysis}

\section{Selection of studies}

Two reviewers conducted database searches in duplicate independently. Titles and abstracts were selected applying inclusion and exclusion criteria. Studies, which were clearly not related to the ONFH and steroid usage, were excluded. The two reviewers independently assessed the full text of the remaining studies for inclusion and exclusion criteria. Inclusion criteria: RCTs, cohort studies and case-control studies, and published between 1985 to 2020. No restriction was placed on the year of publication or language.

Exclusion criteria: Non-human subjects, animal studies, crosssectional studies and case-reports or case-series, studies without control group(s), systematic literature reviews and editorials. Primary studies without full text version and with no response from the correspondence author were also excluded. Discussions between the two reviewers were done for disagreements.

\section{Assessment of methodological quality}

Specific assessment methods and tools to appraise the methodological quality assessment of the included studies according to its nature. For RCTs: Assessment of bias in the included studies fol- lowed the recommendations as described in Chapter 8 of the Cochrane Handbook for Systematic Reviews of Intervention 6.0 [6]. A specific table on the risk of bias assessment was developed according to the above recommendations [29]. The key issues to be addressed are: adequate sequence generation, allocation concealment, blinding, reporting incomplete outcome data (dropouts), no selective reporting, and no potential threats to validity.

For cohort and case-control studies: the Newcastle-Ottawa Scale [26] was used in combination of extra assessment criteria selected by the author. The extra assessment criteria were modified from McLean., et al. [10] which the scoring system was also adopted and modified. The scoring system in current review, with maximum score of 19 , will consider primary studies having over half of the maximum score (i.e. 10 or more out of 19) to be having good quality. A specific form was developed for the two reviewers to assess the quality of the selected primary studies. After the discussions among reviewers to settle the different scorings, the low quality papers might be excluded.

\section{Measures of treatment effect}

The risk ratio (RR) and 95\% confidence interval (CI) were explored for outcomes in RCTs and cohort studies while odds ratio (OR) and 95\% CI for case-control studies. The data from non-randomized studies were more likely to have bias and heterogeneity.

\section{Assessment of heterogeneity}

Heterogeneity of the results has been assessed by visual inspection of the forest plots and by the I2 statistic (test of the heterogeneity). The significance of heterogeneity was re-ported and the potential sources were explored. Appropriate measures, such as "post hoc" analysis, would only be used when necessary.

\section{Subgroup analysis and intervention of heterogeneity}

The subgroup analysis was performed for results with marked heterogeneity when possible subgroups, such as difference in study design, age, gender, race, type of steroid used, dosage, underlying disease for steroid use, methods of measuring the outcomes etc. can be identified.

\section{Sensitivity analysis}

A sensitivity analysis was performed using the criteria for assessment of risk of bias. This was to assess if there is any difference of results in high quality primary studies when comparing with low 
quality ones. The low quality studies, which greatly affect the main effect of the results while unable to explain by subgroup analysis, are excluded.

\section{Results and Discussion}

Results of the search

The initial electronic searches identified 438 literature from the databases and searches through other sources. After checking duplicates, 144 were removed, leaving a total of 294. After reading the titles and abstracts, a further 165 were eliminated after screening, including 29 case-reports, 38 narrative and general reviews, 96 irrelevant topics and 2 without full texts available, leaving a total of 129 for eligibility assessment. A further 124 full-text articles were removed as they failed to fulfill the selection criteria in which 85 articles are not RCT, cohort or case-control studies, 3 citations are prognostic studies, 8 studies were found to have comparison groups of interest but without a placebo group (i.e. a group without steroid exposure), 14 citations have not got relevant data for extraction, 19 citations were excluded after further checking and discussion between the two reviewers to confirm the contents of the studies are irrelevant to this review.

Corresponding authors have been contacted by email upon their email addresses were provided in the articles. However, until the date of report, none of the authors has replied, while one email address was found to be invalid. A total of 5 articles were finally included in the qualitative synthesis. These 5 articles were included in the quantitative synthesis as these included all the data and variables required. The flow chart (Figure 1) shows the process of the study selection.

\section{Included studies}

Five primary studies were included for this review. There are no RCTs, three small sample cohort studies, one case-control study, and one case-control study nested in a survey. They were conducted in Japan [19], the United States [2,9,11] and Canada [28]. All of them were published in English. The summary of the characteristics of the included studies are reported in table 2 below. It includes the information about the methods, participants, interventions, outcomes and risk estimation of the included research.

\section{Characteristics of the participants}

The five studies contained a total of 926 individuals with a mean age of 46.0 (range from $15-82$ ) years ( $71.7 \%$ were male). The de-

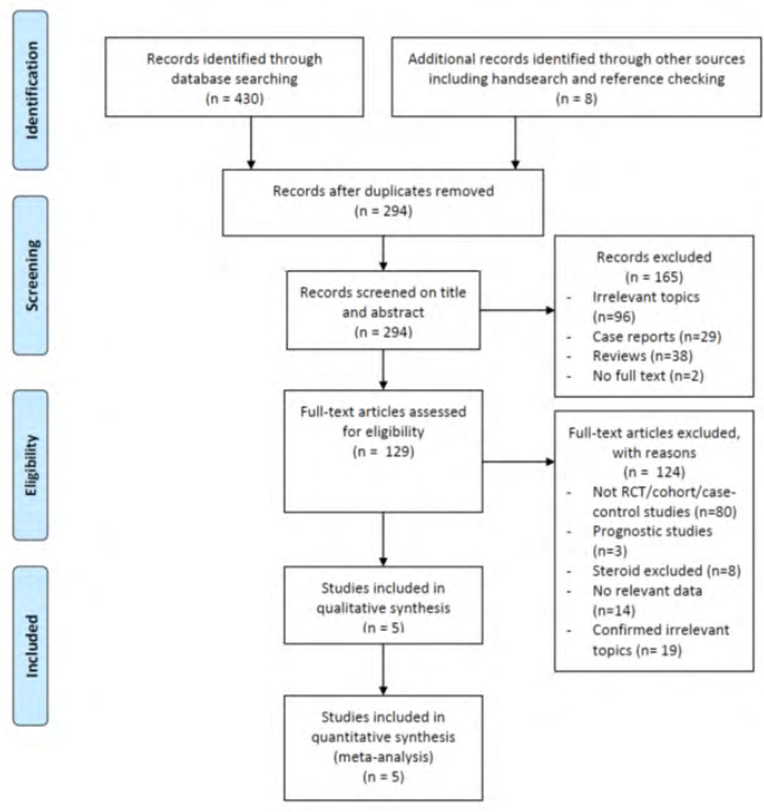

Figure 1: Preferred Reporting Items for Systematic Reviews and Meta-analyses (PRISMA) flow diagram of the search strategy.

tail numbers of participant distribution are provided in Table 2. All participants (including control subjects in case-control studies) in the included studies were non-community samples recruited through hospital or clinic records. The three cohort studies recruited patients with asthma, inflammatory arthritis, chronic pulmonary diseases, and spinal cord injury $[2,9,28]$.

The case-control study nested in a survey [11] recruited HIVinfected participants and a multi-centre case-control study [19] recruited patients from eight orthopaedic surgery departments in Japan with various diseases.

\section{Characteristics of the interventions}

In view of the dosage of steroid used in selected studies, the steroid exposure methods and levels were different among the included studies. The dosage of steroids received by the participants in the two case-control studies have not been specified whilst only the presence or absence of exposure was recorded. 


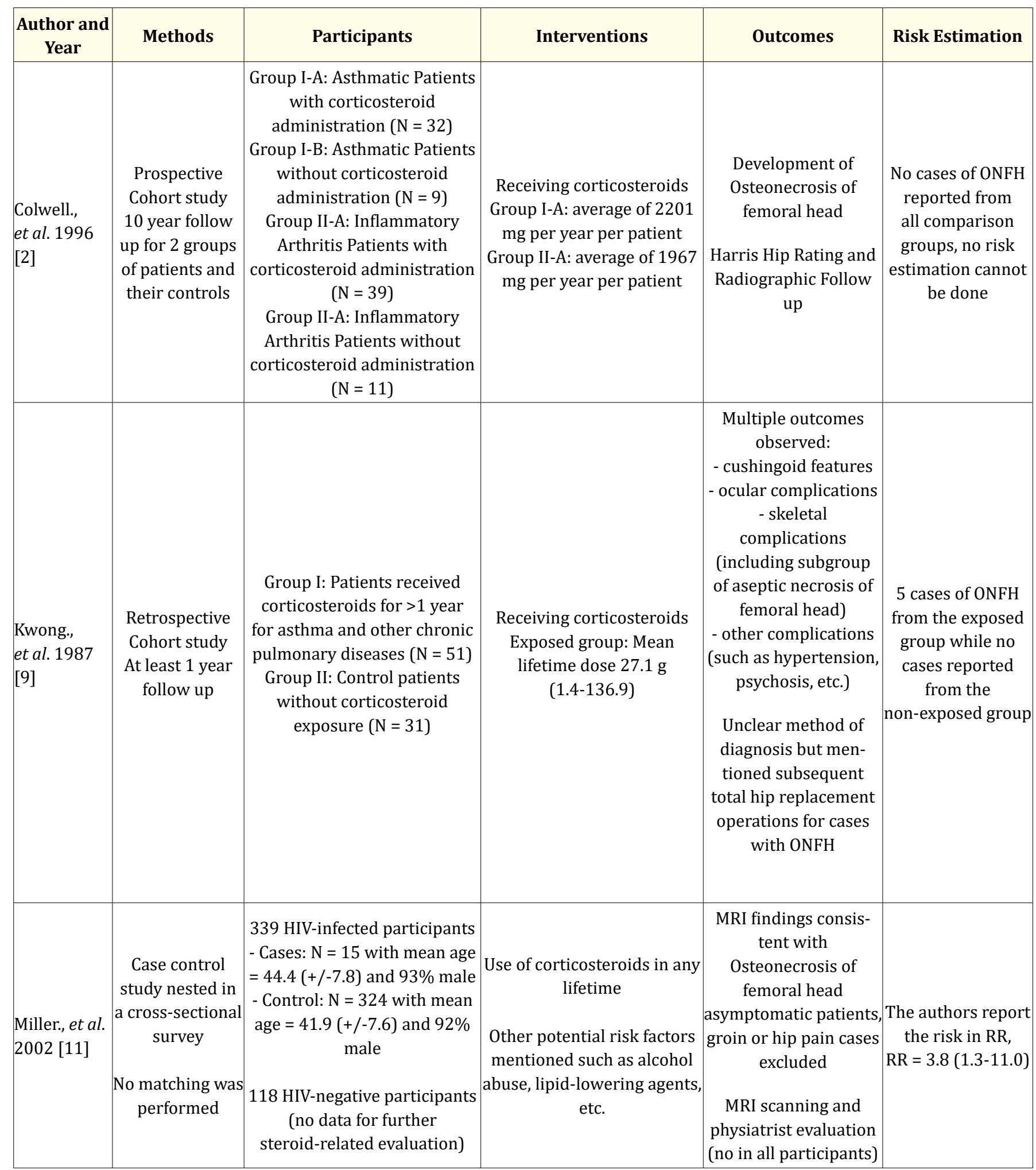




\begin{tabular}{|c|c|c|c|c|c|}
\hline $\begin{array}{l}\text { Sakaguchi., } \\
\text { et al. } 2010 \\
{[19]}\end{array}$ & \begin{tabular}{|} 
Multicenter \\
case-control \\
study \\
Participants \\
matched by sex, \\
age and ethnicity \\
Data collected by \\
mailed \\
questionnaire
\end{tabular} & $\begin{array}{c}\text { All subjects recruited from } 8 \\
\text { departments of orthopedic } \\
\text { surgery in Japan } \\
\text { - Cases: } \mathrm{N}=73 \text { with mean age } \\
=44.0(20-79) \text { and } 63 \% \text { male } \\
\text { - Control: } \mathrm{N}=250 \text { with mean } \\
\text { age }=47.5(18-79) \text { and } 60 \% \\
\text { male } \\
\text { Matching ratio of case to } \\
\text { control from } 1: 1-1: 5\end{array}$ & $\begin{array}{l}\text { Varies risk factors has been } \\
\text { explored: } \\
\text { Oral corticosteroid, } \\
\text { gastrointestinal drugs, } \\
\text { sleeping drugs, vitamin } \\
\text { supplement, drinking and } \\
\text { smoking }\end{array}$ & \begin{tabular}{|} 
Development of \\
osteonecrosis of \\
femoral head \\
Radiological features, \\
bone scan, MRI results, \\
histological features \\
Based on the criteria \\
proposed by Research \\
Committee on the \\
Idiopathic \\
Osteonecrosis of the \\
Femoral Head \\
supported by the \\
Ministry of Health, \\
Labour, and Welfare of \\
Japan
\end{tabular} & $\begin{array}{c}\text { Unadjusted OR = } \\
14.3(6.36-32.2) \\
\text { Adjusted OR = 20.3 } \\
\quad(6.73-61.5)\end{array}$ \\
\hline $\begin{array}{l}\text { Wing., et al. } \\
1998 \text { [28] }\end{array}$ & $\begin{array}{l}\text { Retrospective } \\
\text { Cohort Study } \\
\text { Retrospective } \\
\text { data from } \\
\text { non-exposed } \\
\text { group (not } \\
\text { prospectively } \\
\text { followed) } \\
\text { 6-12 months } \\
\text { follow-up }\end{array}$ & $\begin{array}{c}91 \text { spinal cord injured patients } \\
\text { - Exposed group received } \\
\text { steroids: } \mathrm{N}=59 \text { with mean } \\
\text { age=32 (15-64) and } 5 \text { women } \\
\text { - Non-exposed group: } \mathrm{N}=32 \\
\text { with mean age=34 (16-65) } \\
\text { and } 7 \text { women }\end{array}$ & $\begin{array}{l}\text { Short term megadose } \\
\text { methylprednisolone } \\
\text { treatment following one of } \\
\text { the following } 3 \text { protocols } \\
\text { - received a } 30 \mathrm{mg} / \mathrm{kg} \\
\text { loading dose of Mpred given } \\
\text { over } 15 \mathrm{~min} \text {, followed by a } \\
45 \mathrm{~min} \text { pause before } \\
\text { beginning maintenance by } \\
\text { a } 5.4 \mathrm{mg} / \mathrm{kg} / \mathrm{hr} \text { intravenous } \\
\text { infusion for } 23 \mathrm{hrs} \\
\text { - received a naloxone } 5.4 \\
\text { mg/kg bolus with a } 4 \mathrm{mg} / \\
\mathrm{kg} / \mathrm{hr} \text { infusion for } 23 \mathrm{hrs} \\
\text { - received a } 30 \mathrm{mg} / \mathrm{kg} \mathrm{bolus} \\
\text { followed by a } 5.4 \mathrm{mg} / \mathrm{kg} / \mathrm{hr} \\
\text { Mpred intravenous infusion } \\
\text { for } 23 \mathrm{hrs} \text {. }\end{array}$ & $\begin{array}{c}\text { Development of } \\
\text { avascular necrosis } \\
\text { MRI scanning }\end{array}$ & $\begin{array}{l}\text { No cases of ONFH } \\
\text { reported from } \\
\text { all comparison } \\
\text { groups, no risk } \\
\text { estimation cannot } \\
\text { be done }\end{array}$ \\
\hline
\end{tabular}

Table 2: Characteristics of included studies.

The study from Colwell., et al. [2] included patients having continuous (routine usage), intermittent (short-term intra-articular injection) and burst (short term large doses) administration of corticosteroids [2]. The average steroid dosages used were 2201 (range: 53-2012) mg per patient per year for asthmatic patients and 1967 (range: 8-1936) mg per patient per year for inflammatory arthritic patients during the 10 years of follow-up. Participants from the study of Kwong., et al. [9] received mean lifetime dose of corticosteroids of 27.1 (range: 1.4-136.9)g in 7 (range: 1 -25) years (i.e. average $3871 \mathrm{mg}$ per patient per year). The steroid used in the study of Wing., et al. [28] was described in more detail.

Naloxone and methylprednisolone were used in several treatment protocols (see de-tails from the table of characteristics of included studies) with $97.4-154.0 \mathrm{mg} / \mathrm{kg}$ steroid administration per patient in 24 hours, whilst the treatment started within 8 hours of spinal cord injury. 


\section{Characteristics of the outcomes}

The development of ONFH was at least one of the outcomes in all five included studies. Miller., et al. [11] only focused on the asymptomatic type of ONFH. Except Kwong., et al. [9] which has not been specified in detail, X-ray or MRI findings were the major methods used to confirm the outcomes of ONFH.

\section{Methodological quality assessment}

For the quality assessment of the included studies, the risk of bias in included studies was evaluated. NOS was used to assess the risks of biases of the included cohort and case-control studies.

\section{Cohort studies}

Findings of the quality assessment of the included cohort studies are shown in table 3 .

- Subject selection: Among the three cohort studies, all subjects were from hospital sampling with most are historical control from the hospital records. The sample size of the cohorts were small, especially the control (non-exposed) groups were below 50 subjects. All exposure information collected was based on medical records.

- Comparability: Only Kwong., et al. [9] mentioned controlling for age range during non-exposed group subject selection. However, none of these three cohorts had mentioned any statistical adjustments on the confounding factors.

- Outcome: Radiographic films, Harris Hip Rating scale, and MRI scan were used for determining the outcome in two cohorts. Unclear diagnostic method was mentioned in the study by Kwong., et al. [9] while all cases were subsequently required total hip replacement surgery [2,9]. Only Colwell., et al. [2] followed the participants over 10 years and reported the high loss of follow-up rate while the other two cohorts only followed the subjects for about one year.

\begin{tabular}{|c|c|c|c|c|c|}
\hline \multirow[b]{2}{*}{ Study ID } & \multicolumn{3}{|c|}{ NOS for cohort studies } & \multirow[b]{2}{*}{$\begin{array}{l}\text { Other Assessment } \\
\text { items (max:10) }\end{array}$} & \multirow[b]{2}{*}{$\begin{array}{l}\text { Overall score } \\
\text { (max:19) }\end{array}$} \\
\hline & $\begin{array}{l}\text { Selection } \\
\text { (max:4) }\end{array}$ & $\begin{array}{c}\text { Comparability } \\
\text { (max:2) }\end{array}$ & $\begin{array}{l}\text { Outcome } \\
\text { (max:3) }\end{array}$ & & \\
\hline Colwell 1996 & $* * *$ & - & $* *$ & $* * * * * *$ & 11 \\
\hline Kwong 1987 & $* *$ & $*$ & - & $* *$ & 5 \\
\hline Wing 1998 & $* *$ & - & $*$ & $* * * *$ & 7 \\
\hline
\end{tabular}

Table 3: Summary table of methodological quality assessment: Cohort Studies.

\section{Case-control studies}

Findings about the quality assessment of the included case-control studies are shown in table 4.

- Subject selection: All subjects (both case and controls) in the two included studies were from hospital/clinic or from the hospital records. The sample sizes were relatively larger. Radiographic features, bone scan results, histological features and MRI scan $[19,28]$ were used for determining the outcome.

- Comparability: Sakaguchi., et al. [19] matched the controls by sex, age group, and ethnicity (only Japanese) but not in fixed proportion while statistical adjustment for other medical or behavioral confounders was reported. The cases and controls from the study by Miller., et al. [11] were not matched and without any statistical adjustment preformed.
- Exposure: The exposure information collected was based on self-administered questionnaires. Non-response rate only mentioned in the study by Sakaguchi., et al. [19].

Three out of five studies scored less than 10 points, which can be regarded as very low quality $[9,11,28]$. Colwell., et al. [2] study scored 11/19, which could be considered as low-medium quality while Sakaguchi., et al. [19] study scored 14/19 could be regards as medium to high quality study. The comparability seems to be one of the critical problems among the low quality studies. This indicates the results obtained from these low quality papers are particularly questionable and thus inconclusive.

\section{Effects of interventions}

The summary of the findings of the analyses of the main comparisons about the effects of interventions is shown in table 5 be- 


\begin{tabular}{|l|c|c|c|c|c|}
\hline \multirow{2}{*}{ Study ID } & \multicolumn{3}{|c|}{ NOS for case-control studies } & \multirow{2}{*}{$\begin{array}{c}\text { Other Assessment } \\
\text { items (max:10) }\end{array}$} & $\begin{array}{c}\text { Overall score } \\
\text { (max:19) }\end{array}$ \\
\cline { 2 - 6 } & $\begin{array}{c}\text { Selection } \\
\text { (max:4) }\end{array}$ & $\begin{array}{c}\text { Comparability } \\
\text { (max:2) }\end{array}$ & $\begin{array}{c}\text { Exposure } \\
\text { (max:3) }\end{array}$ & $*$ & $*$ \\
\hline Miller 2002 & $* *$ & - & $* *$ & $* * * * * * *$ & 4 \\
\hline Sakaguchi 2010 & $* * *$ & $* *$ & $*$ & 14 \\
\hline
\end{tabular}

Table 4: Summary table of methodological quality assessment: Case-control Studies.

low. Owing to different statistical methods for cohort and case-control studies, sub-group analyses were performed although there was only one outcome of interest in this review.

\begin{tabular}{|l|c|c|c|c|}
\hline $\begin{array}{c}\text { Outcome or } \\
\text { Subgroup }\end{array}$ & Studies & Participants & $\begin{array}{c}\text { Statistical } \\
\text { Method }\end{array}$ & $\begin{array}{c}\text { Effect } \\
\text { Estimate }\end{array}$ \\
\hline $\begin{array}{l}\text { Development } \\
\text { of ONFH } \\
\text { (cohort } \\
\text { Studies) }\end{array}$ & 3 & 264 & $\begin{array}{c}\text { Risk Ratio } \\
\text { (M-H, Fixed, } \\
95 \% \mathrm{CI})\end{array}$ & $\begin{array}{c}7.45 \\
{[0.40,} \\
139.58]\end{array}$ \\
\hline $\begin{array}{l}\text { Exposure } \\
\text { of steroids } \\
\text { (case-control } \\
\text { studies) }\end{array}$ & 2 & 662 & $\begin{array}{c}\text { Odds Ratio } \\
\text { (M-H, Ran- } \\
\text { dom, 95\% CI) }\end{array}$ & $\begin{array}{c}7.05 \\
{[2.19,} \\
22.67]\end{array}$ \\
\hline
\end{tabular}

Table 5: Summary table about data analyses of the main comparisons about the effects of interventions.

For the cohort studies, the forest plot (Figure 2) showed that the relative risk of the pooled data is 6.77 with $95 \%$ CI $(0.39$ to 118.36). This indicates when compared to people having steroid usage to those do not have steroid exposure, no statistically significant differences in the risk for the development of osteonecrosis of femoral head. However, since no relative risk can be calculated from Colwell., et al. [2] and Wing., et al. [28] which both reported no development of osteonecrosis of femoral head in both exposed and non-exposed groups [2,28], the final result only reflected the results from the study of Kwong., et al. [9]. Thus, there is not enough information for heterogeneity assessment to be performed [9].

For case-control studies, since the heterogeneity of studies is high (I-square=69\%), random-effect model was adopted in the analysis. The forest plot (Figure 3) showed the pooled data from the 2 case-control studies, pooled odd ratio is 7.05 with $95 \% \mathrm{CI}$ (2.19 to 22.67) which is statistically significant. As there are only

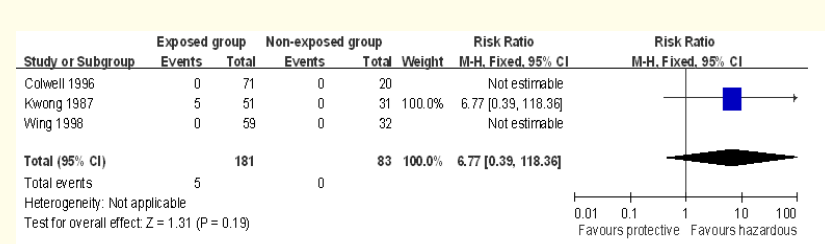

Figure 2: Forest plot for 3 cohort studies (Fixed effect model).

*Fixed effect model used since only one primary study able to contribute to the relative risk calculation.

two included studies in this analysis with high heterogeneity in results, performing sensitivity analysis will not yield meaningful result for interpretation.

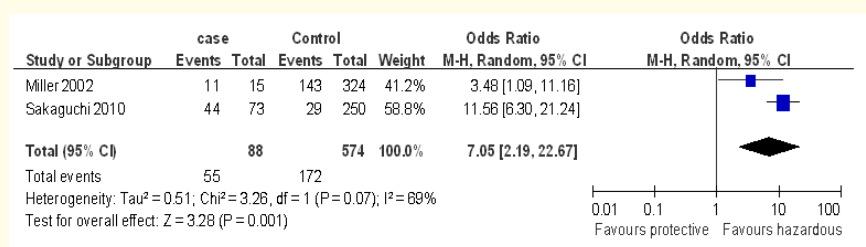

Figure 3: Forest plot for 2 case-control studies (Random effect model).

*Random effect model used since large heterogeneity of the primary studies $(I 2=69 \%)$.

\section{Discussion}

Summary of main results

This review is the first systematic review with meta-analyses attempt to find the risk ratio of steroid usage compared with no 
such exposure in the development of ONFH of people at all ages. The current data from the 2 subgroups are similar in risk ratios but inconsistent in confident intervals for the same outcome. Although the results from the two case-control studies both supported the hypothesis of high risk of steroid usage in development of osteonecrosis of femoral head, the small number of studies identified with potential reporting bias should also be considered when interpreting the results.

Out of the three cohorts, two of them have no cases reported with the target outcome in all comparison groups, and one showed statistical insignificance of risk ratio estimation (only 5 cases in the whole cohort). The two studies without reported cases of ONFH were considered not estimable in meta-analysis while they also contribute to the inconsistency of results. These should all be considered whilst interpreting the results. Since cohort studies should be the better design to answer etiological questions, we cannot be able to draw conclusion with such the small sample sizes and poor quality of the included studies.

\section{Overall completeness and applicability of evidence}

Methodological quality

The heterogeneity of the results among the primary studies could be related to quality of the study methodology, biological variations due to study target populations, techno-logical variations of outcome measures/definitions and intervention variations of types/dose of steroids used. The five studies had different target populations, which may differ from symptoms and co-morbidities. Some populations like chronic pulmonary diseases [9] might have higher probability of having other potential risk factors like smoking which may not be well controlled or reported in the study. Miller., et al. [11] had chosen HIV-infected patients as the target population. However, HIV infection alone, anti-retroviral treatment or protease inhibitor treatment could be risk factors for osteonecrosis of femoral head in HIV-infection population [12].

\section{Outcome measures}

Although development of osteonecrosis of femoral head was the common outcome of interest in all five included studies, the definitions of the outcome and the method of diagnosis differed between studies. Kwong., et al. [9] had the least descriptions on the diagnostic method while the use of MRI may not be as popular at $80 \mathrm{~s}$ and thus the authors mentioned the cases all had to go through operations for joint replacement to confirm the presence of outcome. Colwell., et al. [2] only used X-ray films and a clinical scale for diagnosis of the outcome. This may imply that some cases which meeting the definition of outcome of this review were not detected due to the choice of diagnostic method.

In contrast, Miller., et al. [11] only target patients with asymptomatic osteonecrosis of femoral head and excluded all cases with groin or hip pain. This reduction of potential cases may also cause selection bias for the risk ratio estimation.

\section{Dosage-response relationship and types of steroids}

The included clinical studies involved the prescription of different dosages and types of steroids (e.g. oral corticosteroids, intraarticular injection, pulse therapy or routine use, etc.) as the intervention. Hence, the estimation of dose-response relationship from the cur-rent included studies is difficult. Differences in mean dose of steroid used or types of steroid administration again, varied among these studies and difficult for direction comparison. These small sample case-control studies most reported no significant differences in total dose and/or mean dose received by patients. Further studies about the types and dosages of steroids to be used and the risk of ONFH may be clinically meaningful for the prevention of such clinical problem.

\section{Study design and quality of the evidence}

The quality of the related studies are low in general. Many studies about this topic with anecdotal evidence and in poor controls of their quality. In view of the study design of the evidence, there is no relevant RCTs identified. This may be due to the ethical concerns of conducting a research with potential adverse effects using RCTs and the long follow-up period may be needed for the development of ONFH after the initial exposure. The included studies contain three cohorts and two case-control studies. NewcastleOttawa Scale for cohort and case-control studies with ten points assessment items [10] were adopted to form a scale for quality assessment of the studies, which is specific enough to appraise the quality of those kind of included studies. Thus, GRADEpro was not used for this review.

Studies with low score from NOS tended to have low score out of the ten items. The patterns and details of the scoring can be found. 


\section{Potential biases in the review process}

We had tried to maximize the known synonyms for the search keywords, setting without language barrier and types of publication during electronic searches, and emailing corresponding authors for further information to reduce potential biases in literature search. Although no grey literatures/citations were identified from search results, we believe we have tried to minimize the chance of missing relevant studies in the field.

Agreements and disagreements with other studies or reviews

The only identified quantitative review was the study from Felson., et al. done in 1987 [3]. However, it only included evaluation on steroid dose and bolus steroid on the risk of avascular necrosis of bone. It showed similar results as the excluded studies of our current review. Some narrative reviews or systematic literature reviews were found but none of them specified the risk of steroid usage in the development of ONFH [12-15].

\section{Implications for practice}

Although the risk of steroids towards the development of ONFH remains uncertain, the included studies and excluded studies are pointing out that the risk still exists and being co-related to patient co-morbidity, steroid treatment modality and dosage, etc. Therefore, due to various potential of negative effects of using steroids, particularly in long-term, healthcare professionals should carefully prescribe steroids and remind their patients to be aware of the risk of other potential side-effects of using steroids.

Medical practitioners should balance the potential risks and benefits of using steroids therapy according to both of the spirit of evidence-based practice and patient-centered clinical judgement in practice when they prescribe steroids. For the needs of controlling the exacerbation of the negative effects of the inflammatory conditions; including the result of COVID-19 infection, the unique clinical values of steroids should not be overlook by clinicians due to the reference of the uncertain and unconfirmed research reports. Careful assessment, close clinical monitoring and the screening of people in high-risk of developing osteonecrosis should be provided by the medical practitioners if the use of steroids is unavoidable to manage certain diseases and/or clinical conditions; such as $\mathrm{CO}$ VID-19.

Whilst the risk and dose-response relationship cannot be confirmed, adjuvant therapies should be prescribed during steroid treatment period. For instance, bisphosphonates and vitamin $\mathrm{E}$ should also be prescribed to patients; anticoagulants, vasodilators, physical therapy, exercises or combined therapies.

\section{Implications for research}

Literature on the risk of steroid usage to the development of ONFH is constantly growing together with more understanding of the diagnosis and drug effects, and changes in dosages used. This poses the question whether it would be useful to constantly update this topic with different angles to allow more potential relevant comparisons and under-standings of this area or not.

Report of the RECOVERY trial implies the practical value of steroids therapy for the management of clinical conditions due to COVID-19 infection. However, this review shows that clinical studies are still required to clearly understand the risks of developing ONFH from the use of steroids. Steroid therapy still has its clinical value for the management of severe inflammatory conditions including the consequences of COVID-19 in the pandemic. Due to the limited evidences being identified, further researches with large cohort or case-control studies would be useful to determine the risk factors for the development of ONFH. The information from excluded studies also suggested that further researches on treatment modalities like pulse therapy, dosage of steroid usage, or the links between steroid usage and co-morbidity of patients would be useful to help clinicians in making decision on treatments and dosages.

\section{Limitation}

Most of the included studies are low in methodological quality. Thus, a conclusive result of high level of evidence is limited to yield due to many potential biases and unclear information in the study reports. In addition, the focuses of this review are on analyzing the risk effect and the dose-response relationship of using steroids particularly to the development of ONFH (i.e. comparing risk between exposure and non-exposure of steroid). Therefore, studies without a placebo group (non-exposed group) were excluded in this re-view, which may contain information about the adverse effects of steroids usage to the musculoskeletal system.

\section{Conclusion}

To manage COVID-19 and the associated inflammatory conditions due to the infectious conditions, the use of steroids is recommended for the pandemic. Whilst the clinical guideline for the 
suitable prescription of steroids is unclear, the potential risks from the administration of steroids remains a concern in actual practice including the risk of developing ONFH. This systematic review indicates that it is not possible to confirm the use of steroid therapy leads to the consequences of ONFH based on the specific clinical studies. It is inconclusive about whether people received steroids would have higher risk of having ONFH. There are five cohorts and case-control studies could be identified in the specific areas about the use of steroids and the risks of developing ONFH. Studies are mostly heterogeneous. None of the reported studies was randomized. The reporting of confounders and other parameters of lots of the related studies was poor. There are wide differences in relation to the dosages, types and prescriptions among the similar studies. Dose-response relationship of steroid therapy to the relative risk of ONFH remains un-clear. Due to the relatively low quality of the studies, it is not possible to draw a confirmed conclusion from the related evidence regarding to the relationship of the use of steroids and the risk of developing ONFH.

Similar risk ratios favouring steroids usage being associated to the development of ONFH were found from the sub-group analyses of the studies identified in this review. There are differences in their confidence intervals. The heterogeneous results from these primary studies and the potential biases from two not estimable studies will need to be considered when interpreting the results. As the quality of the included studies is generally low, current available evidence is heavily limited. The development of ONFH from using steroids is still inconclusive, as the risk remains uncertain from current evidence.

Whilst the risk of using steroids for developing ONFH cannot be confirmed, the unique clinical values of steroids should not be overlook due to the reference of the uncertain and unconfirmed research reports. Steroids therapy remains its values patients such as those infected with COVID-19 in the pandemic. To minimize the possible risks from the use of steroid therapy, it should be administered with caution in clinical practice. Robust research are needed to determine suitable dosage and safe guidelines for using steroids to obtain the best therapeutic effects of this medication to benefit for the mankind.

\section{Acknowledgements}

Nil.

\section{Conflict of Interest}

The authors declare no conflict of interest.

\section{Bibliography}

1. Babis George C., et al. "Osteonecrosis of the Femoral Head". Journal of Orthopedics 34.1 (2011): 39-48.

2. Colwell Clifford W., et al. "Osteonecrosis of the Femoral Head in Patients with Inflammatory Arthritis or Asthma Receiving Corticosteroid Therapy". Journal of Orthopedics 19.11 (1996): 941-946.

3. Felson DavidT and Jennifer Anderson. "Across-Study Evaluation of Association between Steroid Dose and Bolus Steroids and Avascular Necrosis of Bone". The Lancet 329.8538 (1987): 902-906.

4. Ficat RP. "Idiopathic Bone Necrosis of the Femoral Head. Early Diagnosis and Treatment". The Journal of Bone and Joint Surgery. British volume 67.1 (1985): 3-9.

5. Gardeniers JWM., et al. "The Arco Staging System: Generation and Evolution since 1991". Osteonecrosis. Springer (2014): 215-218.

6. Higgins Julian PT., et al. Cochrane Handbook for Systematic Reviews of Interventions. John Wiley and Sons, (2019).

7. Hirota Yoshio., et al. "Association of Alcohol Intake, Cigarette Smoking, and Occupational Status with the Risk of Idiopathic Osteonecrosis of the Femoral Head". American Journal of Epidemiology 137.5 (1993): 530-538.

8. Kubo Toshikazu., et al. "Clinical and Basic Research on SteroidInduced Osteonecrosis of the Femoral Head in Japan”. Journal of Orthopaedic Science 21.4 (2016): 407-13.

9. Kwong, FK., et al. "Corticosteroid Complications in Respiratory Disease”. Annals of Allergy 58.5 (1987): 326-30.

10. McLean, Sionnadh Mairi., et al. "Risk Factors for the Onset of Non-Specific Neck Pain: A Systematic Review”. Journal of Epidemiology Community Health 64.7 (2010): 565-72.

11. Miller Kirk D., et al. "High Prevalence of Osteonecrosis of the Femoral Head in Hiv-Infected Adults”. Annals of Internal Medicine 137.1 (2002): 17-25. 
12. Mont Michael A., et al. "Nontraumatic Osteonecrosis of the Femoral Head: Ten Years Later". JBJS 88.5 (2006): 1117-1132.

13. Mont Michael A., et al. "Systematic Analysis of Classification Systems for Osteonecrosis of the Femoral Head". JBJS 88 (2006): 16-26.

14. Mont Michael A., et al. "The Natural History of Untreated Asymptomatic Osteonecrosis of the Femoral Head: A Systematic Literature Review". JBJS 92.12 (2010): 2165-2170.

15. Myers Thomas G., et al. "Outcomes of Total Hip Arthroplasty for Osteonecrosis of the Hip: Systematic Review and MetaAnalysis". Journal of Current Orthopaedic Practice 21.1 (2010): 81-88.

16. NHS. "Corticosteroids". (2011).

17. P Grant. "Coumarin Anticoagulants and Endocrine Interactions". Coumarin Anticoagulant Research Progress Ed. Edardes, J. New York: Nova Science Publishers (2008). 11-45.

18. Prescott Hallie C and Todd W Rice. "Corticosteroids in Covid-19 Ards: Evidence and Hope During the Pandemic". JAMA 324.13 (2020): 1292-1295.

19. Sakaguchi Motonobu., et al. "Impact of Oral Corticosteroid Use for Idiopathic Osteonecrosis of the Femoral Head: A Nationwide Multicenter Case-Control Study in Japan". Journal of Orthopaedic Science 15.2 (2010): 185-191.

20. Singh Awadhesh Kumar., et al. "Role of Corticosteroid in the Management of Covid-19: A Systemic Review and a Clinician's Perspective". 14.5 (2020): 971-78.

21. Steinberg David R and Marvin E Steinberg. "The University of Pennsylvania Classification of Osteonecrosis". Osteonecrosis. Springer (2014). 201-06.

22. Steinberg, M.E. “Osteonecrosis". The Merck Manuals March 2008 (2008).

23. Steinberg Marvin E and David R Steinberg. "Classification Systems for Osteonecrosis: An Overview”. Journal of Orthopedic Clinics 35.3 (2004): 273-83.

24. Sugano Nobuhiko., et al. "The 2001 Revised Criteria for Diagnosis, Classification, and Staging of Idiopathic Osteonecrosis of the Femoral Head". Journal of Orthopaedic Science 7.5 (2002): 601-05.

25. Wang Ao., et al. "The Pathogenesis of Steroid-Induced Osteonecrosis of the Femoral Head: A Systematic Review of the Literature". Journal of Gene 671 (2018): 103-109.

26. Wells, George A., et al. "The Newcastle-Ottawa Scale (Nos) for Assessing the Quality of Nonrandomised Studies in Meta-Analyses". Oxford (2000).

27. WHO. Corticosteroids for Covid-19: Living Guidance, 2 September 2020: World Health Organization (2020).

28. Wing PC., et al. "Risk of Avascular Necrosis Following Short Term Megadose Methylprednisolone Treatment". Journal of Spinal Cord 36.9 (1998): 633-636.

29. Wong May CM., et al. "Topical Fluoride as a Cause of Dental Fluorosis in Children". Cochrane Database of Systematic Reviews 1 (2010).

30. Zhang Bin and Shuixing Zhang. "Corticosteroid-Induced Osteonecrosis in Covid-19: A Call for Caution". Journal of Bone and Mineral Research 35.9 (2020): 1828-1829.

\section{Assets from publication with us}

- Prompt Acknowledgement after receiving the article

- Thorough Double blinded peer review

- Rapid Publication

- Issue of Publication Certificate

- High visibility of your Published work

Website: www.actascientific.com/

Submit Article: www.actascientific.com/submission.php Email us: editor@actascientific.com

Contact us: +919182824667 